\title{
Comparing the immuno-expression of endothelial markers in normal oral mucosa, oral epithelial dysplasia and oral squamous cell carcinoma: relationship between e-cadherin, vimentin, cd3 I, cd I 7 and epithelial-mesenchymal transition
}

\begin{abstract}
Objective: To evaluate the expression of E-cadherin, Vimentin, CD31, CD117 in Oral Epithelial Dysplasia (OED) and Oral Squamous Cell Carcinoma (OSCC), in comparison with Normal Oral Mucosa (NOM).

Material and Methods: Descriptive case study. Histological samples were analyzed by immunohistochemistry against E-cadherin, Vimentin, CD31, CD117. E-cadherin expression was evaluated by extent and intensity, Vimentin was evaluated by intensity and CD31 and C117 quantitatively.

Results: The extent of E-cadherin was greater than $75 \%$ in all NOM samples, $81 \%$ of the OED samples and $47 \%$ of the OSCC samples. The intensity of E-cadherin was high in NOM $(100 \%)$ and in OSCC (36\%). Mean CD31 was $21.2 \pm 5$ in NOM samples, $23.4 \pm 5.8$ in OED samples and $36.7 \pm 10.8$ in OSCC samples. Mean CD117 was $7.2 \pm 2.5$ in NOM samples, $12.8 \pm 5.3$ in OED samples and 22.7 \pm 5.3 in OSCC samples.

Conclusion: The molecular marker E-cadherin is significantly more down-regulated in dysplastic cells in comparison to NOM cells, while the mesenchymal marker Vimentin is expressed by epithelial cells in samples of NOM, OED and OSCC; questioning their value as prognostic markers. Finally, the quantification of CD31 and CD117 increased significantly in OSCC samples.
\end{abstract}

Keywords: mouth/oral neoplasms, epithelial mesenchymal transition, immunohistochemistry, pathological neovascularisation, case series
Volume 4 Issue 3 - 2017

Alejandra Fernández,' Maureen Marshall,' Natalia Santibañez,' Carolina Martínez, ${ }^{1,2}$ Javier Fernández, ${ }^{2}$ René Martínez,' Jorge Briceño, ${ }^{1,3}$ Consuelo Zumaran, ${ }^{3,4}$ Juan Pablo Peñaloza, ${ }^{3,4}$ Ignacio Fuentevilla, ${ }^{3,4}$ Ziyad S Haida ${ }^{1,3-6}$

'Facultad de Odontología, Universidad Andrés Bello, Chile ${ }^{2}$ Facultad de Odontología, Pontificia Universidad Católica, Chile 'BioMAT'X, Centro de Investigación Biomédica, Universidad de los Andes, Chile

${ }^{4}$ Plan de Mejoramiento Institucional (PMI) en Innovación I+D+i, Universidad de los Andes, Chile

${ }^{5}$ Facultad de Medicina, Universidad de los Andes, Chile

${ }^{6}$ Facultad de Odontología, Universidad de los Andes, Chile

Correspondence: Ziyad S Haidar, Implantologist (Cert Implantol), Oral and Maxillofacial Surgeon (MSc OMFS), FRCS (Canada), FACS, MBA, PhD, Professor and Scientific Director, Faculty of Dentistry, Universidad de Los Andes, Santiago de Chile, Founder and Head of BioMAT'X, Biomedica Research Center (CIB), PMI I+D+i, Department for Research, Development and Innovation, Universidad de Los Andes, Mons. Álvaro del Portillo 12.455 - Las Condes, Santiago, Chile,Tel +56226181372, Fax +56222149468, Email zhaidar@uandes.c

Received: July 15, 2017 | Published: September 25, 2017
Abbreviations: bFGF, basic fibroblast growth factor; $\mathrm{CC} 1$, cell conditioning solution-1; DNA, deoxyribo-nucleic acid; E-cadherin, epitheia-cadhein; EMT, epithelial-mesenchymal transition; IHC, inmuno-histochemistry; IL-4, Interleukin-4; IL-6, interleukin-6; NOM, normal oral mucosa; OED, oral epithelial dysplasia; OSCC, oral squamous cell carcinoma; PDGF, platelet-derived growth factor; $\mathrm{SCF}$, stem cell factor; TGF- $\beta$, transforming growth factor-beta; VEGF, vascular epidermal growth factor

\section{Introduction}

Epithelial cancers mostly affect the oral cavity with 9 out of 10 oral neoplasms diagnosed and classified as oral squamous cell carcinoma (OSCC). ${ }^{1,2}$ The mechanism ${ }^{3}$ by which a normal or oral dysplastic epithelium becomes tumorigenic and invasive is multifactorial, however it is widely-accepted that epithelial mesenchymal transition (EMT), angiogenesis and inflammation, play a role. Indeed, EMT is a process by which an epithelial cell undergoes biochemical changes to assume a mesenchymal phenotype, ${ }^{4}$ which confers a greater capacity to migrate and invade anatomic sites far away from the site of origin. ${ }^{5}$

The epithelial markers identified during EMT associated with tumor progression are E-cadherin and Vimentin. ${ }^{6,7}$ Briefly, E-cadherin is an important calcium-dependent transmembrane glycoprotein (cell adhesion molecule and signal transduction factor) located in the epithelial tissue. It can direct the formation of protein complexes attached to the actin cytoskeleton in combination with $\beta$-catenin formation which can prevent and reduce tumor cell adhesion. Hence, E-cadherin is an important symbol of occurrence of the loss of EMT. On the other hand, Vimentin is a cytoskeletal protein, not expressed in normal epithelial cells. It is widely distributed in fibroblasts, endothelial cells, and lymphocytes in the interstitial cells. The abnormal expression of Vimentin was also observed in a variety of epithelial tumors, and had close relationship with differentiation, invasion and metastasis of cancer cell. Here in, the role of angiogenesis in early stages of carcinogenesis is still unclear. Yet, several research 
groups $^{8-11}$ describe an increase in angiogenesis in the malignant transformation of oral epithelium into OSCC.

In malignant neoplasms, the regulation of angiogenesis is lost, resulting in an increase in pro-angiogenic factors and/or loss of inhibitory angiogenic factors, ${ }^{12}$ changing the balance in favor of tumor angiogenesis. Mast cells favor angiogenesis in OSCC by releasing pro-angiogenic factors such as VEGF, bFGF, histamine. ${ }^{13}$

To study angiogenesis and mast cells in OSCC, it is common to quantify the immunoexpression of CD31 (platelet endothelial cell adhesion protein) and CD117 (receptor tyrosine kinase protein/protooncogene c-Kit) respectively. ${ }^{13,14}$ Therefore, in this descriptive study (of a human case series) we evaluate the expression of E-cadherin, Vimentin, CD31, CD117 in OED and OSCC, in comparison with NOM.

\section{Materials and methods}

\section{Sample collection}

All samples were obtained via the anatomical pathology service of the Faculty of Dentistry of the Universidad Andrés Bello inViña del Mar, between 2004 and 2012. Study design was reviewed and approved by the bioethics committee of the Universidad Andrés Bello. Evaluation and statistical analysis was performed at BioMAT'X, Universidad de los Andes.

There were 16 NOM samples histologically diagnosed adjacent to irritative fibroids, vulgar warts, mucoceles, amalgam tattoos and nevi; 16 samples of OED and 19 OSCC samples and 19 NOM samples; 18 samples of OED and 19 OSCC samples were obtained to be used in the analysis by E-cadherin- Vimentin and CD31-CD117, respectively.

The inclusion criteria corresponded to the samples that had the information related to age, gender, diagnosis and anatomical location. The exclusion criteria corresponded to samples that presented artifacts of histological technique or absence of connective tissue in the area adjacent to epithelial dysplasia or OSCC.

\section{Immunohistochemistry}

From each sample, 4 micron thick sections were made. Each sample was mounted on a xilanized slide, de-waxed and rehydrated by immersion in dilutions of ethanol in graduated series. An antigenic recovery process was performed for with $\mathrm{CC1}$ (Cell Conditioning Solution Conditioner-1). Tissue sections were incubated with the anti-E-cadherin monoclonal antibody (DAKO), anti-Vimentin (Ventana), human CD-31 monoclonal antibody (Dako), and human $\mathrm{CD}-117$ monoclonal antibody, (BioSB) to $37^{\circ} \mathrm{C}$. The entire protocol was performed using automated equipment BenchMarck GX (C2014 Roche Products). Finally, the cuts were dehydrated and mounted with coverslips using entellán as a medium of assembly.

\section{Evaluation of samples}

Analysis of samples was performed by two double-blinded oral pathologists, previously standardized and calibrated. All samples were observed under an optical microscope (Olympus ${ }^{\circledR}$ CX-31).

Evaluation of immunostained samples against E-cadherin: Immunopositive epithelial cells for E-cadherin were considered to have a pattern of plasma membrane staining. Determination of the extent of immunostaining for E-cadherin in the epithelial thickness of NOM, OED and OSCC was qualitatively evaluated and categorized into $1=1-25 \% ; 2=26-50 \% ; 3=51-75 \% ; 4=75 \%$. The intensity was evaluated qualitatively in an ordinal way, in 0 : absence of staining;
1+: slight staining; 2+: moderate staining; $3+$ : staining marked. Each sample was compared with negative and positive controls locate on the same slide. The positive control was segment of tonsil and thyroid tumor. The negative control was obtained by omission of the primary antibody.

Evaluation of immunostained samples against vimentin: Immunepositive epithelial cells for Vimentin were considered to have a pattern of cytoplasmic staining. The determination of immunostaining intensity was qualitatively evaluated ordinally at 0 : no staining; 1+: slight staining; 2+: moderate staining; $3+$ : staining marked. Each sample was compared with negative and positive controls locate on the same slide. The positive control was segment of tonsil, liver and serous carcinoma. The negative control was obtained by omission of the primary antibody.

Evaluation of immunostained samples against CD31 and CD117: Immunopositive epithelial cells for CD31 and CD117 were considered to have a pattern of cytoplasmic staining. To determine vascular mean and mast cell mean, for each slide 3 pictures were photographed at a magnification of $40 \mathrm{x}$, using Micrometrics ${ }^{\circledR}$ SE Premium, 5.1 megapixel Vs 2.9 digital camera incorporated into the optical microscope. It was counted any tubular structure coated by endothelial cells, individual endothelial cells or in islets immunoreactive to the CD-31 antibody was considered as a blood vessel. Mean vascular and mean mast cell was determined using the following formula: Number of vessels or mast cell in picture 1+ Number of vessels or mast cell in picture $3+$ No of vessels or mast cell in picture) $/ 3$.

\section{Statistical analysis}

Age was represented by median and interquartile range. Gender, location, extent and intensity were represented by absolute and relative frequency. The Kruskal-Wallis test was used to analyze the extension and/or intensity according to the diagnosis. The post-hoc test of Conover-Iman was used to evaluate the differences in the range average. The comparison of the mean number of vessels and mast cell according to diagnosis was performed using ANOVA, with Bonferroni post hoc test. A significance level of 0.05 was used and statistical analysis was performed with the STATA $12 \AA$ program (StataCorpLP, Texas, USA).

\section{Results}

Data on the number of samples included in the study, age, gender and location for each diagnosis are shown in Table 1. The extent and intensity of E-cadherin expression according to diagnosis is shown in Figure $1 \&$ Table 2. The extent of E-cadherin in NOM, OED and OSCC is different $(p<0.0022)$ with a distinction between NOM and OSCC $(p=0,002)$ and, OED and OSCC $(p=0,0107)$. However, NOM and OED did not show difference $(p=0,07)$. The analysis of E-cadherin intensity according to diagnosis indicates that there are differences between the 3 groups $(\mathrm{p}<0.0001)$. The NOM results differ from OED $(p<0.001)$ and OSCC $(p<0.001)$. However, there were no differences between OED and OSCC $(\mathrm{p}=0.058)$. When correlating the extent and intensity of E-cadherin, a significant positive association was obtained with a Spearman rho of 0.5413. This indicates that increasing the marker's range also increases its intensity.

Intensity expression of Vimentin according to study group is shown in Figure $2 \&$ Table 3 . We found a statistically significant association between intensity and diagnosis $(p<0.0002) .81 .25 \%$ of the NOM samples were negative for the marker, while $37.50 \%$ of the DEO samples presented intensity 2 and $36.84 \%$ of the COCE samples showed intensity 3 . The results obtained differed between 
MON and OED $(\mathrm{p}<0.0203)$, between MON and OSCC $(\mathrm{p}<0.000)$ and between OED and OSCC $(\mathrm{p}<0.0044)$. There is a significant negative association with a Spearman rho of -0.4849 when correlating the intensity of E-cadherin with that of Vimentin, indicating that increasing the intensity of the immunostaining of one of the markers decreases the intensity of the other.

Table I Distribution of the sample by clinical and demographic characteristics

\begin{tabular}{llllll}
\hline Samples & $\begin{array}{l}\text { Number of } \\
\text { samples }\end{array}$ & $\begin{array}{l}\text { Median age } \\
\text { (years) }\end{array}$ & $\begin{array}{l}\text { Frequency of } \\
\text { females\% }\end{array}$ & $\begin{array}{l}\text { Frequency of } \\
\text { males\% }\end{array}$ & $\begin{array}{l}\text { Localization\% } \\
\text { High risk-low risk }\end{array}$ \\
\hline $\begin{array}{l}\text { E-cadherin vimentin } \\
\text { NOM }\end{array}$ & 16 & & & \\
OED & 16 & 22,25 & 68,75 & 31,25 & -- \\
OSCC & 19 & 55,12 & 50 & 50 & $61,11-38,89$ \\
CD3 I, CDII7 & 72,78 & 57,89 & 42,11 & $73,68-26,32$ \\
NOM & 19 & 21,4 & & & \\
OED & 18 & 56,3 & 73,68 & 26,32 & - \\
OSCC & 19 & 72,8 & 50 & 50 & $61,11-38,89$ \\
\hline
\end{tabular}

Table 2 Distribution of E-cadherin extent and intensity according to diagnosis

\begin{tabular}{lllllll}
\hline & \multicolumn{7}{l}{ Intensity } & & & & \\
\cline { 2 - 7 } Extention & $\mathbf{2}$ & $\mathbf{3}$ & $\mathbf{4}$ & $\mathbf{I}$ & $\mathbf{2}$ & $\mathbf{3}$ \\
& $\mathbf{2 6 - 5 0 \%}$ & $\mathbf{5 1 - 7 5 \%}$ & $\mathbf{7 6 - 1 0 0 \%}$ & $\mathbf{M i l d}$ & $\mathbf{M o d e r a t e}$ & $\mathbf{I n t e n s i v e}$ \\
\cline { 2 - 7 } & $\mathbf{n}(\%)$ & $\mathbf{n}(\%)$ & $\mathbf{n}(\%)$ & $\mathbf{n}(\%)$ & $\mathbf{n}(\%)$ & $\mathbf{n}(\%)$ \\
\hline NOM & -- & - & $16(100)$ & -- & -- & $16(100)$ \\
OED & $1(6,25)$ & $2(12,5)$ & $13(81,25)$ & & $10(62,5)$ & $6(37,50)$ \\
OSCC & -- & $10(52,63)$ & $9(47,37)$ & $2(10,53)$ & $13(68,42)$ & $4(21,05)$ \\
\hline
\end{tabular}
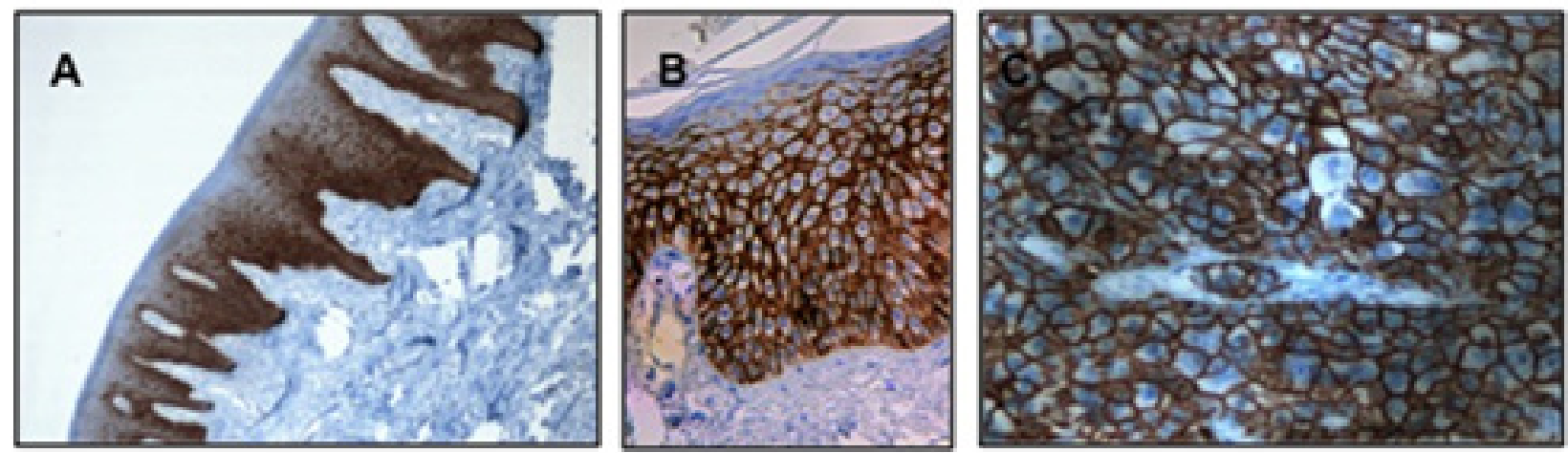

Figure I E-cadherin in A. NOM. B. OED. C. OSCC.
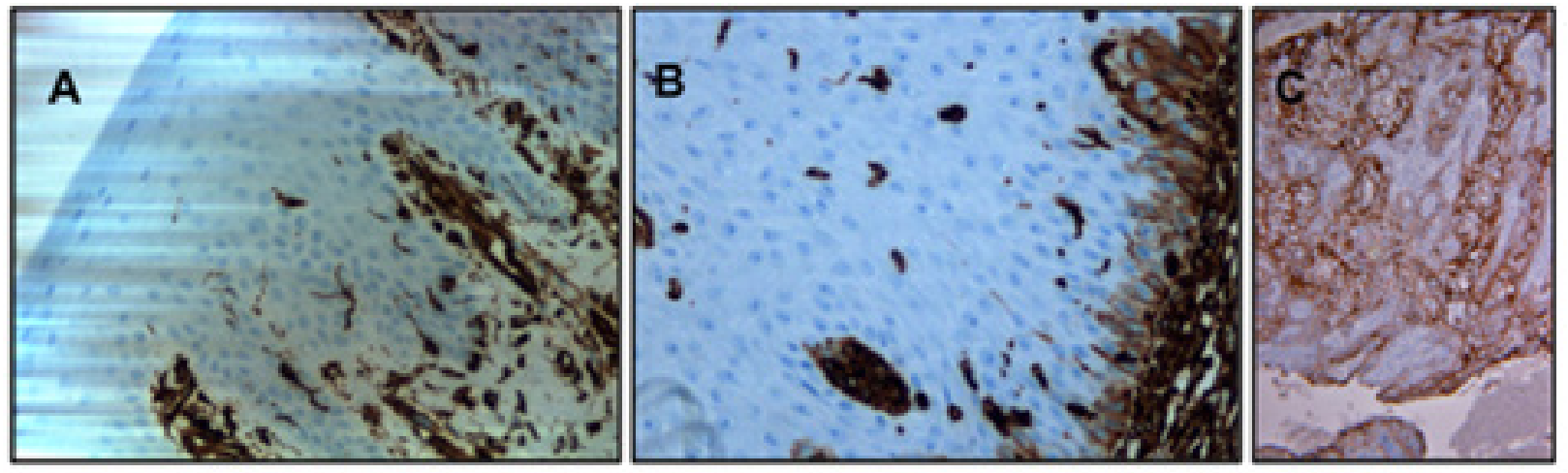

Figure 2 Vimentin in A. NOM. B. OED. C. OSCC.

Citation: Fernández A, Marshall M, Santibañez N, et al. Comparing the immuno-expression of endothelial markers in normal oral mucosa, oral epithelial dysplasia and oral squamous cell carcinoma: relationship between e-cadherin, vimentin, cd3 I, cd I I7 and epithelial-mesenchymal transition. MOJ Anat Physiol. 2017;4(2):30I-306. DOI: 10.15406/mojap.2017.04.00135 
Table 3 Distribution of Vimentin intensity according to diagnosis

\begin{tabular}{lllll}
\hline \multicolumn{1}{l}{ Intensity } & & & \\
\hline & $\mathbf{0}$ & $\mathbf{I}$ & $\mathbf{2}$ & $\mathbf{3}$ \\
\cline { 2 - 5 } & $\mathbf{n}(\%)$ & $\mathbf{n}(\%)$ & $\mathbf{n}(\%)$ & $\mathbf{n}(\%)$ \\
\hline NOM & $13(81,25)$ & - & $3(18,75)$ & -- \\
OED & $6(37,50)$ & $4(25)$ & $6(37,50)$ & -- \\
OSCC & $2(10,53)$ & $5(26,32)$ & $5(26,32)$ & $7(36,84)$ \\
\hline
\end{tabular}

The mean number of vessels and number of mast cells according to diagnosis is shown in Figure 3,4 \& Table 4. The mean number of vessels was different according to diagnosis $(\mathrm{p}<0.001)$. The mean number of vessels was different between NOM and OSCC $(p<0.01)$ and between OED and OSCC $(\mathrm{p}<0.01)$. However, no differences were shown between NOM and OED ( $p=0.91)$. Regarding mean number of mast cell, all groups showed statistically significant differences $(\mathrm{p}<0.001)$. An association was detected by associating the mean density of mast cells with mean vascular density. Both variables showed a statistically significant direct correlation of moderate intensity between both variables $(r=0.48, p<0.001)$.
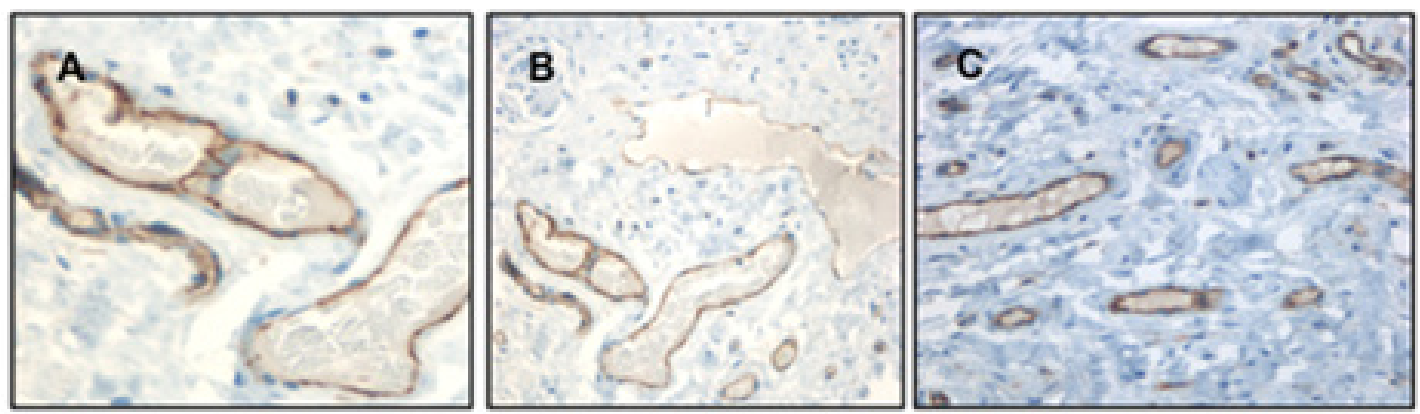

Figure 3 CD3I in A. NOM. B. OED. C. OSCC.
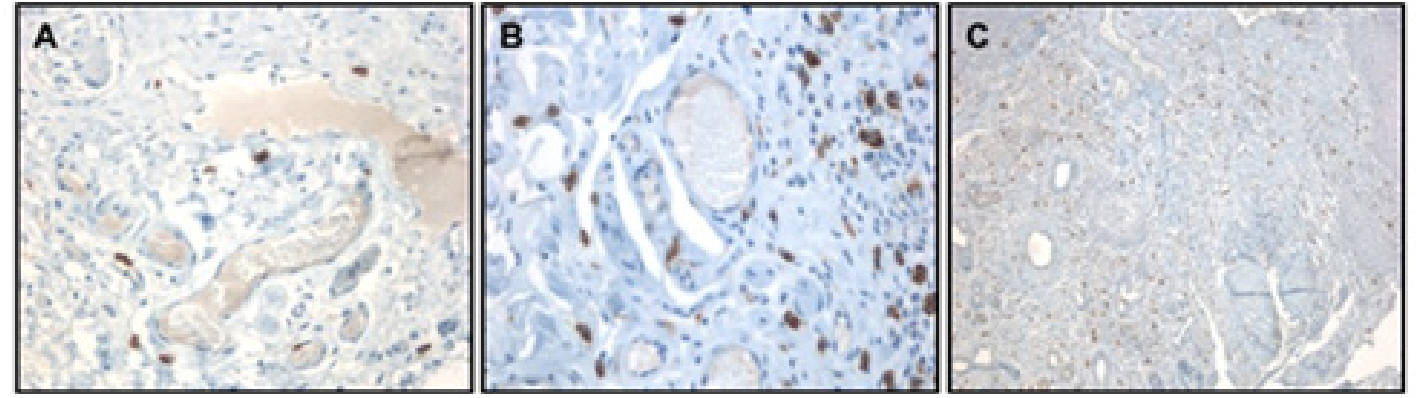

Figure 4 CDI 17 in A. NOM. B. OED. C. OSCC.

Table 4 Mean number of vessels and cell mast according to diagnosis

\begin{tabular}{lllll}
\hline Diagnostic & $\begin{array}{l}\text { Vascular } \\
\text { media } \\
\text { density } \\
\text { (vessels/ } \\
\mathbf{m m}^{2} \text { ) }\end{array}$ & Cl95\% & $\begin{array}{l}\text { Mastocyte media } \\
\text { density(cells/ } \\
\mathbf{m m}^{2} \text { ) }\end{array}$ & Cl 95\% \\
\hline NOM & $21.2 \pm 5.0$ & $18.8-23.6$ & $7.2 \pm 2.5$ & $6.0-8.4$ \\
OED & $23.4 \pm 5.8$ & $20.5-26.3$ & $12.8 \pm 5.3$ & $10.6-14.5$ \\
OSCC & $36.7 \pm 10.8$ & $31.5-41.9$ & $22.7 \pm 5.3$ & $20.1-25.3$ \\
\hline
\end{tabular}

\section{Discussion}

OED is a histopathological term that denotes the presence of alterations in the differentiation or maturation of oral epithelium ${ }^{15,16}$ which is more likely to undergo malignant transformation compared to its normal counterpart. ${ }^{17,18}$ Such alterations seem to be part of chronologically-sequenced morphological changes through which keratinocytes pass during their malignization process. ${ }^{17}$ Often initiated in normal mucosa, then would undergo dysplastic changes, and eventually progress to an OSCC. ${ }^{18,19}$ Therefore, it would be expected to find that $\mathrm{OED}$, as a potentially malignant lesion, affects younger patients than those with OSCC, as our results where the average age of the OED was significantly lower than the present in the cases of OSCC.

In Chile, it has been reported that OED is more frequent in female gender and OSCC in masculine gender. ${ }^{20}$ Unlike our results where we found that OED did not present preference for gender and OED more frequent in women. However, in most countries, men are more exposed to unhealthy lifestyles and carcinogenic factors such as alcohol, tobacco and diet, which have been associated with the pathogenesis of the disease. ${ }^{1,21}$ In general, age groups that were most affected by ODE and OSCC correspond to the 5th and 7th decade, respectively, and coincides with that reported in the literature. ${ }^{22,23}$ This difference could be due to the fact that for the development of an OSCC, the action of carcinogens that provoke non-lethal mutations in the DNA for a long period of time is necessary so that the first cell that multiplies autonomously and uncontrolled will originate into the neoplastic mass. The passage of time leads to an increase in exposure to risk factors, to which additional genetic and epigenetic changes associated with age are added. ${ }^{24}$ It was also observed that the mean age of OEDs was significantly lower than the mean age in OSCC cases, which is consistent if we consider that OED is a potentially malignant lesion that may be the anteroom for the development of an OSCC. $^{20}$ 
Extent and intensity of E-cadherin is higher in NOM, followed by OED and OSCC (Table 2), which may indicate that the cells are in EMT, a fundamental process during oncogenesis where there is a decrease in the expression of the junctional epithelial cells and the tendency to acquire a mesenchymal phenotype that will confer a greater capacity to migrate to distant sites. Similar findings are reported by others ${ }^{25-27}$ where a decreased expression of E-cadherin in tumor cells was detected in comparison to the strong expression of the same molecule in NOM.

Expression of vimentin in epithelium was not identified in most samples of the NOM group. Unlike the MON group, immunoexpression of Vimentin in $37.5 \%$ of samples from the OED group showed moderate intensity, which may be due to a decrease in the expression of epithelial junctions causing partial or total loss of cell polarity, Together with an increase in the expression of the mesenchymal marker, giving rise to cell migration in an irregular pattern without even passing through the basement membrane. In the OSCC samples, we found that the intensity of Vimentin in most cases was marked (36.84\%). The same happens in prostate, gastric, colorectal, mammary, cervical-uterine and COCE carcinomas in reported findings ${ }^{28,29}$ where this over expression is associated with poorly differentiated tumors and an invasive phenotype. This over expression responds to the regulation that undergoes the gene coding for Vimentina, either through transcriptional activators, epigenetic modifications or posttranslational modifications. From this it could be inferred that the intensity investigated for Vimentina in the carcinomas, unlike the groups NOM and OED, would be revealing a process of cellular reprogramming by means of which the neoplastic epithelial cells acquire a mesenquimal phenotype, forcing them to change their morphology and acquire mobility, allowing them to penetrate the basement membrane, invade the subepithelial connective tissue and enter lymphatic or blood vessels.

Angiogenesis is a growing process as malignant transformation of the oral mucosa progresses, probably due to the release of growth factors by tumor, endothelial and inflammatory cells, such as VEGF, bFGF and PDGF..$^{30}$ Our findings, increased number of vessels (Table 4), coinciding with other reports ${ }^{31}$ where vascularization increased between oral lesions presenting with hyperplasia, OED and OSCC. From this it could be inferred that there is a change in the phenotype of OSCC in relation to OED and NOM and strong presence and action of proangiogenic factors on normal endothelial receptors or the presence of numerous mutated endothelial receptors that promote neovascularization in OSCC.

With respect to the CD117 immunoexpression, we noticed that the average number of mast cells increased as a function of the severity of the lesion, the lesion was larger in OSSC than in OED and NOM. Apparently, mast cells would play a role in the progression of healthy oral mucosa affected by mutational changes towards dysplasia and OSCC. This finding is consistent with some of the OSCC literature , $^{8,32-34}$ and contradicts others ${ }^{35,36}$ where the mast cell number showed a linear decrease when studying the same evolutionary process. This fact can be explained by a decrease in the synthesis of chemotactic factors for mast cells, such as SCF, IL-6 and IL- $4{ }^{37}$ and/or an increase in TGF- $\beta$ and INF- $\gamma$ Its apoptosis and decrease in CD117 receptor expression, respectively ${ }^{35-37}$ reflecting a significant modification in the microenvironment during tumor initiation and progression.

\section{Conclusion}

While the quantification of CD31 and CD117 increased significantly in COCE samples, the present study reveals the down- regulation of the molecular marker E-cadherin, suggestive of reduction in dysplastic cells in comparison to NOM cells. An aberrant expression of the mesenchymal marker Vimentin by epithelial cells was also detected in samples of NOM, OED and OSCC. Collectively, our findings further question the value of E-cadherin and Vimentin as prognostic markers.

\section{Acknowledgments}

This work was supported via operating funds provided to BioMAT'X through the Faculty of Dentistry and PMI (Plan de MejoramientoInstitucional), Dirección de Innovación, Universidad de los Andes, Santiago de Chile. The authors also recognize grants from the CONICYT-FONDEF (Código ID16I10366).

\section{Conflict of interest}

The authors declare that they have no conflicts of interest regarding this study.

\section{References}

1. Weatherspoon DJ, Chattopadhyay A, Boroumand S, et al. Oral cavity and oropharyngeal cancer incidence trends and disparities in the United States: 2000-2010. Cancer Epidemiol. 2015;39(4):497-504.

2. Fernández A, Córdova $\mathrm{P}$, Badenier $\mathrm{O}$, et al. Epidemiological characterization of oral cancer. Literature review. J Oral Res. 2015;4(2):137-145.

3. Hanahan D, Weinberg RA. Hallmarks of cancer: the next generation. Cell. 2011;144(5):646-674.

4. Lamouille S, Xu J, Derynck R. Molecular mechanisms of epithelialmesenchymal transition. Nat Rev Mol Cell Biol. 2014;15(3):178-196.

5. Benedetti I, Reyes N. Transición epitelial-mesenquimal en la progresión del adenocarcinoma prostático. Iatreia. 2015;28(4):420-433.

6. Heerboth S, Housman G, Leary M, et al. EMT and tumor metastasis. Clin Transl Med. 2015;4:6.

7. Natarajan J, Chandrashekar C, Radhakrishnan R. Critical biomarkers of epithelial-mesenchymal transition in the head and neck cancers. $J$ Cancer Res Ther. 2014;10(3):512-518.

8. Michailidou EZ, Markopoulos AK, Antoniades DZ. VEGF expression from human dysplastic or malignant oral epithelium may be related to mast cell density and the subsequent angiogenetic phenomena. Int $J$ Oral Maxillofac Surg. 2012;41(12):1467-1473.

9. Astekar M, Joshi A, Ramesh G, et al. Expression of vascular endothelial growth factor and microvessel density in oral tumorigenesis. J Oral Maxillofac Pathol. 2012;16(1):22-26.

10. Abbas NF, Labib El-Sharkawy S, Abdel Monem El-Shaer M, et al. Immunohistochemical study of p53 and angiogenesis in benign and preneoplastic oral lesions and oral squamous cell. Oral Surg Oral Med Oral Pathol Oral Radiol Endod. 2007;103(3):385-390.

11. Teodoro JG, Evans SK, Green MR. Inhibition of tumor angiogenesis by p53: a new role for the guardian of the genome. $J$ Mol Med. 2007;85(11):1175-1186.

12. Pujari R, Vanaki S, Puranik R, et al. Histomorphometric analysis of vascularity in normal buccal mucosa, leukoplakia, and squamous cell carcinoma of buccal mucosa. J Oral Maxillofac Pathol. 2013;17(3):334-339.

13. Gudiseva S, Santosh ABR, Chitturi R, et al. The role of mast cells in oral squamous cell carcinoma. Contemp Oncol. 2017;21(1):21-29.

14. Liu L, Shi GP. CD31: beyond a maker for endothelial cells. Cardiovasc Res. 2012;94(1):3-5. 
15. Izumo T. Oral premalignant lesions: from the pathological viewpoint Int J Clin Oncol. 2011;16(1):15-26.

16. Caldeira PC, Abreu MH, do Carmo MA. Binary system of grading oral epithelial dysplasia: evidence of a bearing to the scores of an immunohistochemical study. J Oral Pathol Med. 2012;41(6):452-453.

17. Shirani S, Kargahi N, Razavi SM, et al. Epithelial dysplasia in oral cavity. Iran J Med Sci. 2014;39(5):406-417.

18. Barnes L, Eveson JW, Reichart P, et al. World Health Organization Classification of Tumours: Pathology and Genetics of Head and Neck Tumours. IARC Press, Lyon, France 2005. p. 1-435.

19. Bouquot JE, Speight PM, Farthing PM. Epithelial dysplasia of the oral mucosa-Diagnostic problems and prognostic features. Curr Diagn Pathol. 2006;12(1):11-21.

20. Martínez C, Hernández M, Martínez B, et al. Frequency of oral squamous cell carcinoma and oral epithelial dysplasia in oral and oropharyngeal mucosa in Chile. Rev Med Chile. 2016;144(2):169-174.

21. Dholam KP, Chouksey GC. Squamous cell carcinoma of the oral cavity and oropharynx in patients aged 18-45years: A case-control study to evaluate the risk factors with emphasis on stress, diet, oral hygiene, and family history. Indian J Cancer. 2016;53(2):244-251.

22. Dost F, Ford PJ, Farah CS. Heightened risk of second primary carcinoma of the head and neck following cervical neoplasia. Head Neck. 2014;36(8):1132-1137.

23. Fitzpatrick SG, Honda KS, Sattar A, et al. Histologic lichenoid features in oral dysplasia and squamous cell carcinoma. Oral Surg Oral Med Oral Pathol Oral Radiol. 2014;117(4):511-520.

24. Feller L, Lemmer J. Oral squamous cell carcinoma: epidemiology, clinical presentation and treatment. J Cancer Ther. 2012;3(4):263-268.

25. Balasundaram P, Singh MK, Dinda AK, et al. Study of b-catenin, E-cadherin and vimentin in oral squamous cell carcinoma with and without lymph node metastases. Diagn Pathol. 2014;9:145.

26. Afrem MC, Margaritescu C, Crait oiu MM, et al. The immunohistochemical investigations of cadherin "switch" during epithelial-mesenchymal transition of tongue squamous cell carcinoma. Rom J Morphol Embryol. 2014;55(3 Suppl):1049-1056.
27. Pereira $\mathrm{CH}$, Morais $\mathrm{MO}$, Martins AF, et al. Expression of adhesion proteins (E-cadherin and b-catenin) and cell proliferation (Ki-67) at the invasive tumor front in conventional oral squamous cell and basaloid squamous cell carcinomas. Arch Oral Biol. 2016;61:8-15.

28. Satelli A, Li S. Vimentin as a potential molecular target in cancer therapy or Vimentin, an overview and its potential as a molecular target for cancer therapy. Cell Mol Life Sci. 2011;68(18):3033-3046.

29. Costa LC, Leite CF, Cardoso SV, et al. Expression of epithelialmesenchymal transition markers at the invasive front of oral squamous cell carcinoma. J Appl Oral Sci. 2015;23(2):169-178.

30. Ribatti D, Crivellato E. Mast cells, angiogenesis, and tumour growth. Biochim Biophys Acta. 2012;1822(1):2-8.

31. Li C, Shintani S, Terakado N, et al. Microvessel density and expression of vascular endothelial growth factor, basic fibroblast growth factor, and platelet-derived endothelial growth factor in oral squamous cell carcinomas. Int J Oral Maxillofac Surg. 2005;34(5):559-565.

32. Motasham N, Babakoohi S, Nejad N. Mast cell density and angiogenesis in oral dysplastic epithelium and low- and high- grade squamous cell carcinoma. Acta Odontologica Scandinavica. 2010;68(5):300-304.

33. Anuharada A, Kiran Kumar Naik B, Vijay Srinivas G, et al. Incidence of mast cells in oral squamous cell carcinoma: a short study. J Oncol. 2014;2014:614291.

34. Telagi N, Ahmed Mujib BR, Kulkarni PG, et al. The master switch: Comparative study of mast cell in oral epithelial dysplasia, oral submucous fibrosis and oral squamous cells carcinoma and their association with inflammation and angiogenesis. J Oral Maxillofac Pathol. 2015;19(1):25-29.

35. Cheema VS, Ramesh V, Balamurali PD. The relevance of mast cells in oral squamous cell carcinoma. J Clin Diagn Res. 2012;6(10):1803-1807.

36. Oliveira-Neto HH, Leite AF, Costa NL, et al. Decrease in mast cells in oral squamous cell carcinoma: possible failure in the migration of these cells. Oral Oncol. 2007;43(5):484-490.

37. Theoharides TC, Conti P. Mast cells: the Jekyll and Hyde of tumor growth. Trends Immunol. 2004;25(5):235-241. 